\title{
(2) Building capacity: getting evidence-based practice into healthcare professional curricula
}

\section{OPEN ACCESS}

Elaine Lehane $\odot,{ }^{1}$ Heloise Agreli, ${ }^{1}$ Simone 0 ' Connor, ${ }^{1}$
Josephine Hegarty, ${ }^{1}$ Patricia Leahy Warren, ${ }^{1}$ Deirdre Bennett, ${ }^{2}$
Catherine Blake,,${ }^{3}$ Frank Burke, ${ }^{4}$ Mark Corrigan, ${ }^{5}$
Jonathan Drennan, ${ }^{1}$ Martina Hayes, ${ }^{4}$ Elizabeth Heffernan,
Frances Horgan, ${ }^{7}$ Helen Lynch, ${ }^{8}$ Joseph McVeigh, ${ }^{8}$
Nicole Müller, ${ }^{8}$ Elizabeth O'Keeffe,,${ }^{9}$ Niamh O'Rourke, ${ }^{10}$
Eve O'Toole, ${ }^{11}$ Colm O'Tuathaigh, ${ }^{12}$ Laura Sahm, ${ }^{13}$
Eileen Savage

10.1136/bmjebm-2020-111385

- Additional material is published online only. To view, please visit the journal online (http://dx.doi.org/ 10.1136/bmjebm-2020111385).

For numbered affiliations see end of article.

Correspondence to: Dr Elaine Lehane, Nursing \& Midwifery, University College Cork, National University of Ireland, Cork, Ireland; e. lehane@ucc.ie

\section{Abstract}

Fostering a culture of clinical effectiveness in healthcare is crucial to achieving optimum outcomes for patients. Evidence-based practice (EBP) is a cornerstone of clinical effectiveness. An EBP capacity-building project commenced in Ireland in 2016, in collaboration with the Centre of Evidence-Based Medicine in Oxford. A key part of this project, reported here, was the development of a competency framework for education in EBP and clinical effectiveness to ensure responsiveness of education standards and curricula of healthcare professionals in this area. Methods Following a review of national and international reports, professional guidance documents and empirical literature pertaining to clinical effectiveness education (CEE), a preliminary competency framework was developed. Stakeholder consultations were conducted over a 6-month period, which consisted of 13 focus groups $(n=45)$ and included representatives from clinical practice, higher education and professional training sectors, regulator/accrediting bodies, the Department of Health (Ireland) and patient/service user groups. Results An overarching interprofessional competency framework for CEE was proposed and included the following domains: EBP, quality improvement processes, implementation strategies and collaborative practice: a total of 16 competencies and 60 indicators.

Conclusion A competency framework for CEE for health and social care professionals is presented. It is intended that this framework will provide guidance to healthcare educators and regulators in the construction and revision of curricula, learning outcomes, teaching and assessment strategies, and graduate/clinician attributes. by BMJ.

To cite: Lehane E, Agreli $\mathrm{H}$, $O^{\prime}$ Connor S, et al. BMJ Evidence-Based Medicine Epub ahead of print: [please include Day Month Year]. doi:10.1136/ bmjebm-2020-111385

\section{Introduction}

Clinical effectiveness is a fundamental approach to improving patient safety and quality in health

\section{Summary box}

What is already known about this subject?

- Evidence-based practice (EBP), a key component of clinical effectiveness, is a fundamental approach to providing high-quality and safe healthcare.

- Competency frameworks, if well developed and contextualised, can provide educators with a curricular blueprint in supporting learners to develop the attributes required of them to ensure effective and efficient healthcare.

\section{What are the new findings?}

- An approach to competency framework development that can guide clinical effectiveness education is described.

- Competency domains, key to informing clinically effective practice, include (1) EBP, (2) quality improvement processes, (3) implementation strategies and (4) collaborative practice.

How might it impact on clinical practice in the foreseeable future?

- Explicit integration of the framework competencies throughout academic and clinical learning domains of health professional curricula is required in order to create frequent, relevant and valued opportunities for students and practitioners to engage in the application of EBPs and clinical effectiveness processes now and into the future. service delivery and promotes healthcare that is 
including evidence-based practice (EBP), clinical guidelines, clinical practice guidance, implementation science and clinical audit. ${ }^{2}$ To ensure that health service users receive effective care, it is recommended that health and social care professions incorporate the necessary knowledge, skills and attitudes encompassed within clinical effectiveness processes into their professional education programmes and registration requirements. ${ }^{2}$

The acquisition of competencies for the application of clinical effectiveness in practice begins during an individual's professional training. To this end, health professional education programmes across the lifelong learning continuum must be designed to target such competencies. ${ }^{2}$ A competency framework can assist education and service providers to develop curricula by making clear the precise knowledge and skills needed in order to provide care. ${ }^{3}$ Competency-based education (CBE) is widely used in both the regulation of practice and curricular design in health and social care professional education. In recent years, CBE for healthcare professionals has been promoted internationally. Some well-known examples include 'Tomorrow's Doctors' (UK), the Canadian Medical Education Directives for Specialists (CanMEDS) Competency Framework ${ }^{5}$ and the Palliative Care Competency Framework $^{6}$ (Europe). However, CBE is not without its critics. It is contended that competency is not synonymous with competence and that competency models which use criterion-referenced approaches do not encourage the 'deep and reflective' engagement required during professional practice-based learning. ${ }^{7}$ Other problematic areas in the operationalisation of competence, particularly when developing frameworks across healthcare professions, include divergent values among multiple stakeholders. Despite these challenges, it is recognised that competency frameworks, if well developed and contextualised, have much to offer educators in planning how best to support health and social care professionals to develop the attributes required of them to ensure effective and efficient care. ${ }^{68}$

An EBP capacity-building project commenced in Ireland in 2016, to build capability and leadership for EBP, with the ultimate goal of improving patient outcomes. A key part of this project was the development of a competency framework for education in EBP and clinical effectiveness, to ensure responsiveness of education standards and curricula of healthcare professionals in this area. Through a creative collaborative approach between national policy makers (Department of Health), academic bodies, researchers, clinical educators, accreditation bodies and professional regulators, in conjunction with ongoing mentorship from the Centre of Evidence-Based Medicine, Oxford, this work seeks to address the lack of standardised EBP and clinical effectiveness education (CEE) and training in a concerted effort to enhance realworld practice and to encourage the next generation of leaders in EBP in Ireland.

\section{Methods}

A two-phase approach to the framework development process was undertaken: a scoping review and stakeholder consultation.

\section{Phase I: scoping review}

This phase of the research systematically mapped and categorised existing competency frameworks classified under the umbrella term 'clinical effectiveness education': two research questions guided the review:

1. What competency frameworks (or guidance documents) of CEE currently exist?

2. What are the competencies aligned to clinical effectiveness?

\section{Inclusion criteria}

- Studies with a principal focus on competency frameworks or curriculum development processes of clinical effectiveness or components thereof (eg, EBP, implementation science (IS) and quality improvement (QI)).

Papers were excluded if they

- Described competency frameworks specific to health conditions and clinically effective care.

- Focused on theoretical reviews of different components of clinical effectiveness.

- Contained narratives, letters, discussion papers and/or opinion pieces reporting competency frameworks for CEE.

\section{Search strategy}

An extensive search on the EBSCO (Elton B. Stephens Co.) database, incorporating CINAHL, MEDLINE, Academic Search Complete, PsycARTICLES, Psychology and Behavioural Science Collection and ERIC, was conducted. The search strategy employed the following keywords: undergraduate OR postgraduate OR graduate OR (subject, MH) "Health personnel" OR "health Practitioners" OR "medical personnel” AND "clinical effectiveness" OR "implementation science" OR (subject, $\mathrm{MH}$ ) "Evidence based practice" OR "Evidence based medicine" OR "Quality of healthcare" OR "Quality control of medical care" OR "Quality of healthcare" OR "Translational Medical Research" OR "Translational research" AND competenc* OR curricul* OR framework OR (subject, MH) "Competency based education" OR "outcome based education" OR "Competency based teacher education". Publications were limited to those written in English, French, Spanish and Portuguese and published from 2008 to 2018. A grey literature search was performed and included Open Grey, WHO, NHS Evidence database, NICE, UK Evidence for Policy and Practice Information and Co-ordinating Centre, and a list of websites pertaining to thirdsector education in the UK (provided by an expert in the field of healthcare education). In addition, relevant national reports ${ }^{9-11}$ pertaining to EBP and clinical effectiveness were sourced.

\section{Review process}

All potentially eligible papers identified were exported to EndNote V.7.0, where duplicates were removed. Remaining references were then transferred to an Excel 16.0 file with articles screened initially by title and abstract independently (HA). Agreement by two members of the team (HA and EL) was required for an article to merit a full-text read.

\section{Data extraction}

The review questions guided the data extraction process, including details of the components of competency frameworks (or guidance documents) of CEE and competencies described. Empirical literature data were extracted by HA and a sample (20\%) was cross-checked by EL to ensure consistency. Grey literature data were extracted by HA and similarly a sample cross-checked by EL and SOC.

The integration and synthesis of data from international empirical and grey literature led to the generation of a preliminary competency framework.

\section{Phase II: stakeholder consultation}

For phase II, an emergent-systematic focus group design was adopted, wherein, the term 'emergent' refers to the initial focus groups that were used for exploratory purposes and 'systematic' refers to subsequent groups used for verification purposes. ${ }^{12}$ The 
specific aims were to (1) elucidate participants' perspectives on CEE competencies for healthcare professionals and (2) examine proposed competencies and associated indicators of CEE for relevancy, clarity and comprehensiveness.

A purposive sampling strategy was used to ensure participation from a range of stakeholders with vested interest in CEE. Invitations were issued to representatives from:

- Irish higher education institutes providing healthcare profession education (medicine, nursing, midwifery, dentistry, pharmacy, physiotherapy, psychology, occupational therapy, speech and language therapy, podiatry and radiography).

- Professional training, regulatory and accreditation bodies.

- Members of the National Clinical Effectiveness Committee (NCEC) and subgroups.

- Health Service Executive, Health Information and Quality Authority, Higher Education Authority, Health Research Board and patient/service users.

Given the specialised nature of the topic area, it is recommended $^{13}$ to over-recruit between $20 \%$ and $50 \%$ of the total number of respondents required; therefore, 128 invitations were issued.

In preparation for the consultation, an information leaflet that provided background context to the project was sent 1 week prior to the meeting date. The session began with a 20 min presentation which outlined the scope of the project, the findings of the review work completed and the specific objectives. The consultation groups were limited to four participants as recommended by Krueger and Casey ${ }^{14}$ and Morgan ${ }^{13}$ in instances where group members have specialised knowledge and/or experiences to discuss. The sessions were facilitated by a moderator who used a topic guide in conjunction with a copy of the individual domains originating from the completed review. Participants were directed to individually review the draft list of domains, competencies and indicators, following which guided discussion on suggested changes was conducted. The focus groups ended with a debriefing session in which the moderator surmised key points of discussion.

Note-based analysis, guided by an iterative constant comparison method, ${ }^{15}$ was used and included analysis of notes from the focus group, the debriefing session and summary comments from the moderator. While the focus groups were audio-recorded, the transcripts were primarily used to verify/clarify points of discussion. Five of the research team participated in data analysis (EL, HA, SOC, JD and PLW), with two of the researchers (EL and HA) ensuring consistency in the analysis processes. As focus group data were analysed one focus group at a time and were iterative in nature, the analysis of multiple focus groups served as a proxy for theoretical sampling and assisted the researchers in reaching data saturation.

\section{Results}

\section{Phase I}

Thirty-five publications were included for review (21 empirical papers and 14 grey literature documents; see figure 1).

of the 35 publications, no competency framework (or guidance document) pertaining specifically to CEE was found. Frameworks $(n=9)$ and guidance documents $(n=26)$ which focused on individual components of CEE (ie, EBP, QI and IS) were identified. These predominantly originated from the USA, UK, Canada and Australia within the disciplines of nursing and medicine and were applied at postgraduate level. In the majority of cases, the frameworks were discipline-specific, but a number promoted interprofessionalism within the competencies proposed.

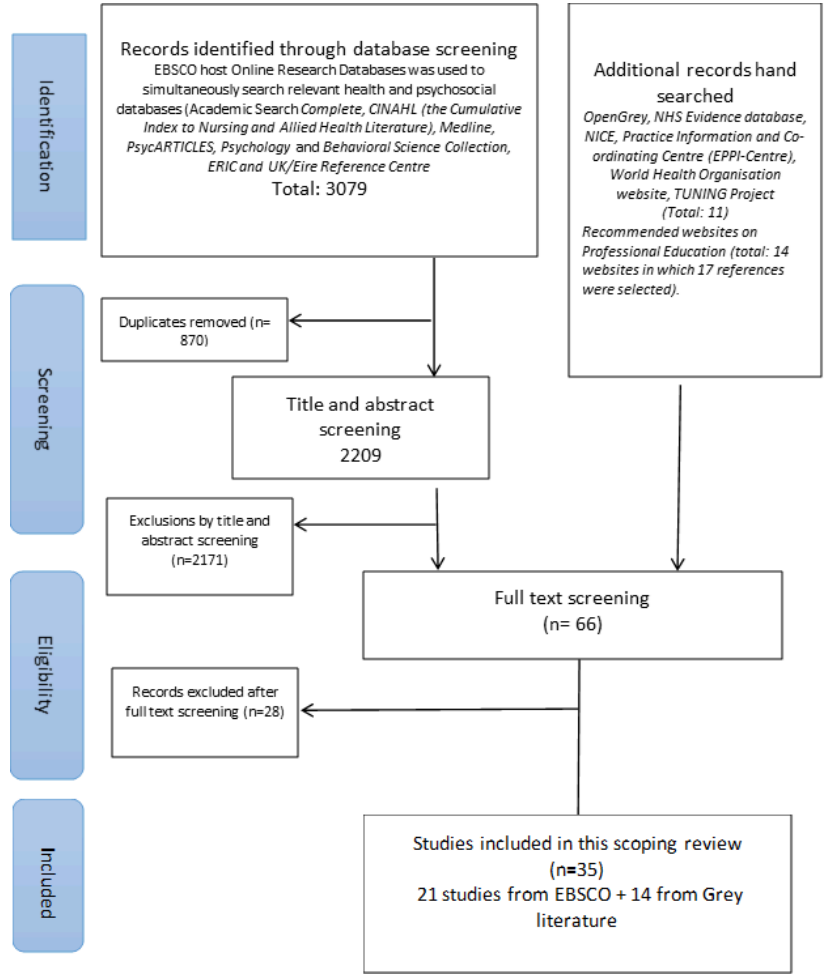

Figure 1 Preferred Reporting Items for Systematic Reviews and MetaAnalyses flow diagram.

Competencies aligned to clinical effectiveness included EBP, QI, implementation strategies and 'related' competencies of collaboration, communication and leadership. Identified competencies within the empirical and grey literature were predominantly from the field of EBP ( $n=28)$, reflecting the trending of EBP in research on CEE. Within the QI $(n=23)$ and IS domains $(n=17)$, the competencies reported were mainly referred to as 'introductory', that is, fundamental concepts associated with QI and implementation processes. Related competencies $(n=29)$ for clinical effectiveness were identified, with the most frequently reported being collaborative practice (teamwork), followed by communication and leadership.

Based on the integration of empirical and grey evidence from this review, four preliminary domains of competencies for CEE were compiled: (1) EBP (five competencies, 25 indicators); (2) QI processes (four competencies, 10 indicators); (3) implementation of clinical effectiveness into practice (three competencies, 9 indicators); and (4) professional practice in the context of clinical effectiveness (three competencies, 11 indicators) (see online supplementary data S1 for preliminary domains and competencies).

\section{Phase I}

Thirteen focus groups were held with 45 stakeholder participants in phase II. The participant profile is presented in table 1 .

General overall feedback deemed the framework relevant, appropriate and important for developing workforce capacity in clinical effectiveness. Participants were satisfied that the domains reflected essential core competencies and that the substantive content of the domains did not overlap. Table 2 outlines the four agreed domains and their competencies.

Feedback specific to each competency domain was also sought. In relation to domain $1-E B P$, which was adopted from Albarqouni et al's ${ }^{16}$ international consensus statement for EBP, 


\begin{tabular}{|c|c|c|c|}
\hline Variable & Subgroup & Respondents (n) & Respondents (\%) \\
\hline \multirow{5}{*}{$\begin{array}{l}\text { Years of experience in } \\
\text { current role }\end{array}$} & $\ll 2$ & 4 & 9 \\
\hline & $3-5$ & 9 & 20 \\
\hline & $6-10$ & 5 & 11 \\
\hline & $>10$ & 23 & 52 \\
\hline & Not specified & 3 & 7 \\
\hline \multirow[t]{3}{*}{ Gender } & Male & 8 & 18 \\
\hline & Female & 33 & 75 \\
\hline & Not specified & 3 & 7 \\
\hline \multirow[t]{7}{*}{ Employer } & Health Service Executive & 12 & 27 \\
\hline & Department of Health & 7 & 16 \\
\hline & Higher education institution & 14 & 32 \\
\hline & Professional body/organisation & 8 & 18 \\
\hline & Health information quality authority & 1 & 2 \\
\hline & Government agency & 1 & 2 \\
\hline & Other & 3 & 7 \\
\hline \multirow{12}{*}{$\begin{array}{l}\text { Health and social care } \\
\text { profession }\end{array}$} & Nursing & 10 & 23 \\
\hline & Midwifery & 2 & 5 \\
\hline & Medicine & 8 & 18 \\
\hline & Physiotherapy & 1 & 2 \\
\hline & Speech and language therapy & 2 & 5 \\
\hline & Pharmacy & 5 & 11 \\
\hline & Dietitian & 1 & 2 \\
\hline & Dentistry & 2 & 5 \\
\hline & Clinical psychology & 1 & 2 \\
\hline & Ophthalmology & 1 & 2 \\
\hline & Other & 13 & 30 \\
\hline & Not specified & 1 & 2 \\
\hline \multirow[t]{6}{*}{ Predominant role } & $\begin{array}{l}\text { Oversight/regulation/standard setting for student/trainee } \\
\text { education }\end{array}$ & 12 & 27 \\
\hline & $\begin{array}{l}\text { Provision of education to students/trainees within a third-level } \\
\text { institution }\end{array}$ & 14 & 32 \\
\hline & Provision of education within a clinical setting & 10 & 32 \\
\hline & $\begin{array}{l}\text { Provision of education from a professional body training } \\
\text { perspective }\end{array}$ & 11 & 25 \\
\hline & Other* & 17 & 39 \\
\hline & Not specified & 3 & 7 \\
\hline
\end{tabular}

*Other: strategic planning and development for nursing and midwifery, policy development, delivery of Continuing Professional Development system for pharmacists, regulation of medicines, building capacity in implementation science, representing Irish Council of General Practitioners on European Committees of Family Medicine and Physician Health, conducting National Healthcare Audit, implementation of standards in disability services, quality and safety management and clinical assessment in health products regulations.

all six competencies ('introductory', 'ask', 'acquire', 'appraise and interpret', 'apply' and 'evaluate') and associated indicators were supported without amendment.

For domain 2-QI processes, four competencies ('introductory', 'appraise', 'engage' and 'reflect') were endorsed with three additional indicators introduced (see online supplementary data S2). In relation to the first competency, introductory, comments received referred to the use of principles and methods of QI rather than including specific examples, for example, the 'plan-do-studyact' cycle. The appraise competency was deemed relevant overall; however, comments from the participants highlighted that competencies should prepare learners to critically appraise information about outcomes of care and not merely identify information. For the engage competency, the importance of patients/service users in the QI cycle was emphasised as requiring explicit recognition within competency indicators. Comments in relation to the reflect

\begin{tabular}{llll}
\hline Table 2 & Final framework domains and competencies for clinical effectiveness education & \\
\hline Domain & Evidence-based practice & Quality Improvement processes & Implementation strategies \\
\hline Competencies & Introductory & Introductory & Collaborative practice \\
& Ask & Appraise & Appraise and Engage \\
& Acquire & Engage & Reflect \\
& Appraise and Interpret & Reflect & \\
& Apply & & \\
& Evaluate & & \\
& &
\end{tabular}


competency stressed that a reflective process should ultimately result in improved quality of care with the need for learners/practitioners to also respond appropriately to reflection outcomes.

There was consensus across all groups that domain 3-implementation strategies was fundamental to supporting learners to effectively implement strategies for integrating evidence into practice. It was commented, across groups, that the content within this domain is an emerging and 'evolving' area, which is not as prominent as EBP or QI within professional education. Three competencies were supported (introductory, appraise and engage and reflect) with two additional indicators proposed. In relation to the introductory competency, several comments highlighted the need to focus on core principles of IS as it specifically contributes to clinical effectiveness. The appraise and engage competency indicators were deemed relevant, with the importance of measurement and evaluation of implementation strategies and the extent to which they impact on practice emphasised. In relation to the final competency, reflect, it was suggested that an indicator promoting 'action on' such reflection was required.

The final domain was renamed collaborative practice in the context of clinical effectiveness and retained its three competencies of interprofessional 'collaboration', 'communication' and 'leadership' with 11 indicators. Participants commented that collaborative practice is essential, alongside interprofessional education, to ensure implementation of clinically effective care. It was suggested that the communication competency be broadened to include the importance of listening and being attentive to patients' insights, concerns and expectations. Participants further suggested the inclusion of one indicator, addressing the need for awareness of individual, collective and professional perspectives that influence one's behaviour and decisions in the context of the collaboration competency. In relation to the final competency leadership, it was suggested that, in addition to 'understanding' leadership principles, a separate indicator was also required that recognised the importance of different leadership styles and the need for commitment from health and social care professionals to support clinical effectiveness.

The amended competency framework was presented to the NCEC subgroup for education and training for final review. Two recommendations were made, with the first advocating for the adoption of a circular structure for displaying the domains to emphasise the importance of integrating all areas of competency for clinically effective practice. The configuration also serves to illustrate that the three domains of EBP, QI processes and implementation form an integrated whole, supported and influenced by collaborative practice (figure 2). The second recommendation highlighted the importance of contextual issues, such as regulatory requirements and educational standards, national healthcare policy or professional setting, that may need to be taken into consideration when applying the framework.

\section{Discussion}

The framework that emerged from this process describes an approach to competency development that can guide CEE. Four competency domains highlight the knowledge and skills that shape judgements essential for clinically effective practice and include (1) EBP, (2) QI processes, (3) implementation strategies and (4) collaborative practice.

This competency framework shares similarities with existing frameworks but also has some important distinctions. Of note, for the EBP domain, we adopted (with permission) the international EBP consensus statement and core competencies derived from Albarquouni et al's ${ }^{16}$ Delphi study. For the QI processes domain,

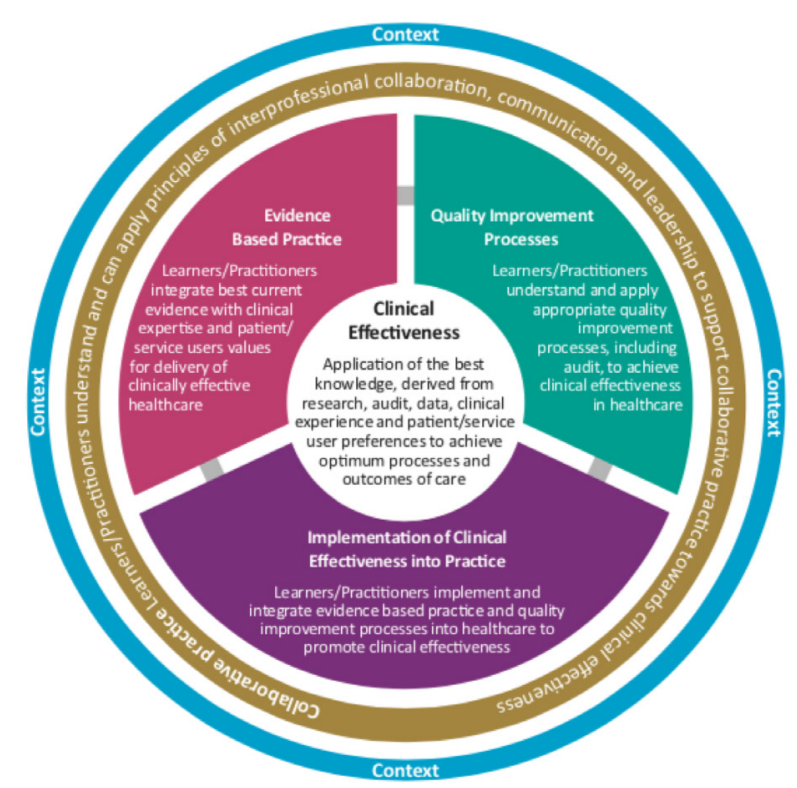

Figure 2 Diagrammatic representation of competency framework for clinical effectiveness education.

overlap with a number of frameworks, most notably, CanMEDS, ${ }^{17}$ the General Medical Council 2009 and 2017 frameworks, ${ }^{418}$ and the Australian Commission on Safety and Quality in Health Care $2010,{ }^{19}$ is evident. Key frameworks and guidance documents used to inform the implementation strategies domain included Gonzales et al's $^{20}$ work on training in implementation and dissemination science and Galbraith et al's ${ }^{21}$ competency framework for a 'realworld' approach to evidence-based medicine for general practitioners. These three domain competencies align to various steps in the EBP process, that is, introductory, appraise, engage/apply and the reflective element of evaluate. The adoption of this 'lens' serves to highlight the variety of stages and contexts required to attain requisite knowledge and skills to provide clinically effective care.

The competencies encompassed within the collaborative practice domain, that is, collaboration, communication and leadership, were common across competency frameworks and guidance documents specific to both professional education frameworks; for example, the GMC, and also in those frameworks focusing on individual components of clinical effectiveness; for example, IS. Due to the cross-cutting nature of this domain and associated competencies, they are conceptualised as fundamental to CEE through their overarching influence on, and support of, EBPs, QI processes and implementation strategies.

This competency framework has been designed for application by a variety of stakeholders/learners in a variety of healthcare contexts. The framework is not a curriculum for CEE; rather, it is a resource which can inform curricular content, teaching, learning and assessment strategies. The framework provides direction for the achievement of competencies at an individual level; therefore, in its application, consideration must be given to the previous CEE and experience of the learner. This, in turn, will impact the level at which the competency is attained. As an underpinning goal of the framework is to educate for clinical effectiveness in workplace practice, attainment of competencies is best supported by practice-based learning activities in the context of the learner's current work or professional environment. 
Although qualitative approaches are often used in identifying and affirming professional competencies, ${ }^{22}{ }^{23}$ such approaches preclude firm conclusions as they rely on group opinions, limited sample sizes and moderator validation bias especially in the a priori presentation of a preliminary framework. In addition, patient/service user representation was absent despite invitations issued. Notwithstanding these limitations, the two-phase approach undertaken facilitated a multifaceted and evidence-based understanding of requirements for CEE. The preliminary framework was informed by a comprehensive scoping review; this approach provided a synthesis of trending topics in empirical and grey literature central to CEE. All competencies were reviewed and refined by individuals with specific expertise from different health and social care professions and settings, affording further credibility to the proposed framework. Debriefing sessions at the end of each focus group in which key points of discussion were presented and feedback was invited as to their accuracy attempted to limit the impact of potential moderator bias. Application and evaluation of the framework, particularly from the patient/service user and international stakeholder perspective, are the next steps required to further validate this framework.

\section{Conclusion}

This competency framework serves as a resource for educators, practitioners and regulators focusing on future health and social care professionals within undergraduate education and qualified health and social care professionals at postgraduate level. It can assist educators in designing, reviewing and standardising curricular content, learning outcomes, and teaching and assessment activities in relation to the four core competency domains of EBP, QI processes, implementation strategies and collaborative practice. There is an increasing awareness and requirement by regulators to ensure competencies for clinical effectiveness are demonstrated as part of licensing and maintenance of licensing for health and social care professionals. The framework will be useful in determining how to guide members to integrate such competencies into professional standards. The explicit integration of the framework competencies throughout academic and clinical learning domains of health professional curricula is required in order to create frequent, relevant and valued opportunities for students and practitioners to engage in the application of EBPs and clinical effectiveness processes now and into the future.

\footnotetext{
Author affiliations

${ }^{1}$ Catherine McAuley School of Nursing \& Midwifery, University College Cork National University of Ireland, Cork, Ireland

${ }^{2}$ Medical Education Unit, University College Cork National University of Ireland, Cork, Ireland

${ }^{3}$ School of Public Health, Physiotherapy and Sports Science, University College Dublin, Dublin, Ireland

${ }^{4}$ School of Dentistry, University College Cork National University of Ireland, Cork, Ireland

${ }^{5}$ Cork Breast Research Centre, Cork University Hospital Group/University College Cork, Cork, Ireland

${ }^{6}$ Nursing and Midwifery Planning and Development Unit, Kerry Centre for Nurse and Midwifery Education, Cork/Kerry, Ireland

${ }^{7}$ School of Physiotherapy, Royal College of Surgeons in Ireland, Dublin, Ireland

${ }^{8}$ School of Clinical Therapies, University College Cork National University of Ireland, Cork, Ireland

${ }^{9}$ Symptomatic Breast Imaging Unit, Cork University Hospital Group, Cork, Ireland

${ }^{10}$ Health Information and Quality Authority, Cork, Munster, Ireland

${ }^{11}$ National Cancer Control Programme, Health Service Executive, Dublin, Ireland
}

${ }^{12}$ School of Medicine, University College Cork National University of Ireland, Cork, Ireland

${ }^{13}$ School of Pharmacy, University College Cork National University of Ireland, Cork, Ireland

Acknowledgements The authors thank the participants of the consultation groups who provided excellent insights and varied perspectives on clinical effectiveness education, and Dr Albarqouni and colleagues for permission to adopt their consensus statement on EBP core competencies. Special thanks to the National Clinical Effectiveness Committee (Education Sub-Group), Department of Health, for guidance throughout the project.

Contributors Conceived and designed the study: EL, JH, NOR and EOT. Scoping review procedures (including data screening, extraction and synthesis): EL, HA and SOC. Clinical teaching expertise and focus group recruitment: MC, COT, HL, NM, FB, MH, DB, CB, EH, FH, JMcV, NOR and LS. Focus group data collection and analysis: PLW, HA, EL, JD and SOC. Research report appraisal, drafting and dissemination: All. Manuscript appraisal and final approval: All.

Funding This work was commissioned by the Clinical Effectiveness Unit, Department of Health, Ireland.

Competing interests NOR was employed by the Department of Health at time of funding.

Patient consent for publication Not required.

Ethics approval Social Research Ethics Committee of University College Cork (ref: Log 2018-045).

Provenance and peer review Not commissioned; externally peer reviewed.

Data availability statement Data are available upon reasonable request. Deidentified participant data are available upon reasonable request from the corresponding author (e.lehane@ ucc.ie). No commercial reuse of data.

Open access This is an open access article distributed in accordance with the Creative Commons Attribution Non Commercial (CC BY-NC 4.0) license, which permits others to distribute, remix, adapt, build upon this work non-commercially, and license their derivative works on different terms, provided the original work is properly cited, appropriate credit is given, any changes made indicated, and the use is non-commercial. See: http://creativecommons.org/licenses/by-nc/4.0/.

ORCID iD

Elaine Lehane http://orcid.org/0000-0002-0144-7467

\section{References}

1 Department of Health (DOH). National Clinical Effectiveness Committee Annual report. Dublin: DOH, 2016.

2 Thomas A, Saroyan A, Dauphinee WD. Evidence-Based practice: a review of theoretical assumptions and effectiveness of teaching and assessment interventions in health professions. Adv Health Sci Educ Theory Pract 2011;16:253-76.

3 Antonoff MB, Swanson JA, Green CA, et al. The significant impact of a competency-based preparatory course for senior medical students entering surgical residency. Acad Med 2012;87:308-19.

4 General Medical Council (GMC). Tomorrow's doctors; outcomes and standards for undergraduate medical education, 2009. Available: https:// www.gmc-uk.org/-/media/documents/tomorrows-doctors-supplementaryguidance_pdf-81919983.pdf [Accessed 13 Mar 2020].

5 Frank JR, Danoff D. The CanMEDS initiative: implementing an outcomesbased framework of physician competencies. Med Teach 2007;29:642-7. 
6 Gamondi C, Larkin P, Payne S. Core competencies in palliative care: an EAPC white paper on palliative care education: Part 2. Eur J Palliat Care 2013;20:140-5.

7 Talbot M. Monkey see, monkey do: a critique of the competency model in graduate medical education. Med Educ 2004;38:587-92.

8 Pijl-Zieber EM, Barton S, Konkin J, et al. Competence and competencybased nursing education: finding our way through the issues. Nurse Educ Today 2014;34:676-8.

9 Department of Health. National Clinical Effectiveness Committee - Annual report. Dublin: DOH, 2015.

10 Department of Health. National Clinical Effectiveness Committee - Annual report. Dublin: DOH, 2017.

11 Lehane E, Warren PL, O'Riordan C, et al. Research on teaching of evidence based practice in Ireland. Report prepared for the clinical effectiveness unit, department of health, Ireland, 2017. Available: https://www.gov.ie/ pdf/?file=https://assets.gov.ie/11518/0a1797901aa640ec8ef0e2f876d4b791. pdf\#page $=1$ [Accessed 13 Mar 2020].

12 Onwuegbuzie AJ, Dickinson WB, Leech NL, et al. A qualitative framework for collecting and analyzing data in focus group research. Int J Qual Methods 2009;8:1-21.

13 Morgan DL. Focus groups as qualitative research. Qualitative Research Methods Series, 1997: 16.

14 Krueger RA, Casey MA. Focus groups. A practical guide for applied research. Thousand Oaks: Sage, 2014.

15 Straus SE, Brouwers M, Johnson D, et al. Core competencies in the science and practice of knowledge translation: description of a Canadian strategic training initiative. Implement Sci 2011;6:127.
16 Albarqouni L, Hoffmann T, Straus S, et al. Core competencies in evidencebased practice for health professionals: consensus statement based on a systematic review and Delphi survey. JAMA Netw Open 2018;1:e180281.

17 Frank JR, Snell L, Sherbino J. The CanMEDS 2015 Physician Competency Framework. Royal College of Physicians and Surgeons of Canada, 2015.

18 General Medical Council. Generic professional capabilities framework, 2017. Available: https://www.gmc-uk.org/-/media/documents/genericprofessional-capabilities-framework-0817_pdf-70417127.pdf [Accessed 13 Mar 2020].

19 Australian Commission on Safety and Quality in Health Care. The National safety and quality health service (NSQHS) standards, 2010. Available: https://www.safetyandquality.gov.au/our-work/assessment-tothe-nsqhs-standards/ [Accessed 13 Mar 2020].

20 Gonzales R, Handley MA, Ackerman S, et al. A framework for training health professionals in implementation and dissemination science. Acad Med 2012;87:1-8.

21 Galbraith K, Ward A, Heneghan C. A real-world approach to evidencebased medicine in general practice: a competency framework derived from a systematic review and Delphi process. BMC Med Educ 2017;17:78

22 Czabanowska K, Klemenc-Ketis Z, Potter A, et al. Development of a competency framework for quality improvement in family medicine: a qualitative study. J Contin Educ Health Prof 2012;32:174-80.

23 Sadeghi-Ghotbabadi F, Shakibazadeh E, Omidvar N, et al. Consensus development on the essential competencies for Iranian public health nutritionists. Public Health Nutr 2015;18:752-8. 